\title{
INVESTIGACION
}

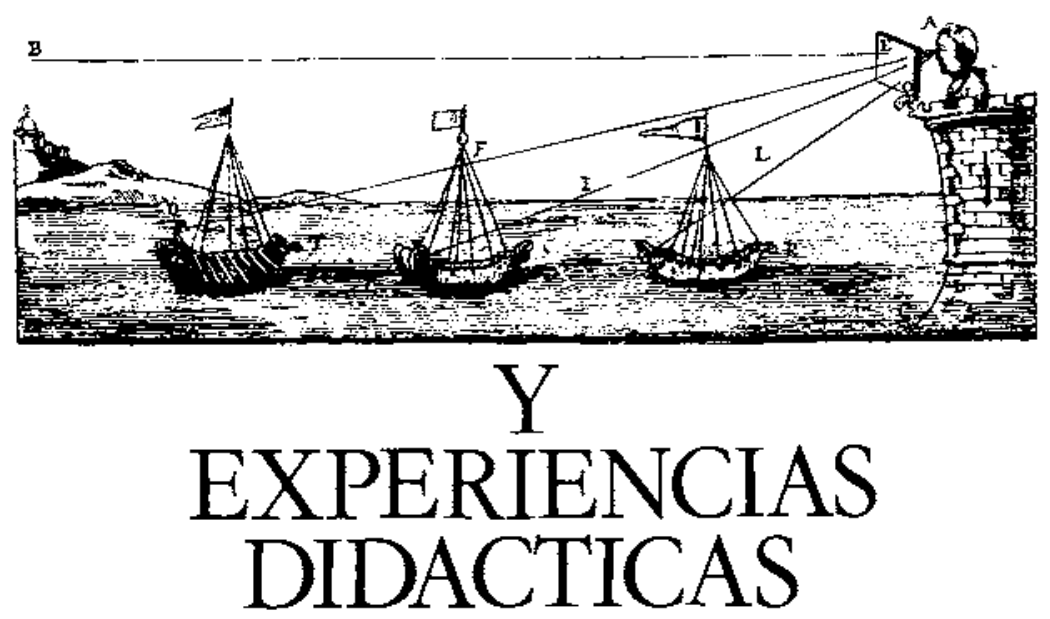

\section{LA FUNCIÓN DEL LENGUAJE EN UN ENFOQUE CONSTRUCTIVISTA DEL APRENDIZAJE DE LAS CIENCIAS}

\author{
LLORENS, J.A. ', DE JAIME, $M^{\mathrm{a}} \mathrm{C}^{{ }^{2}}$ y LLOPIS, R. ${ }^{3}$ \\ (1) Centro de Profesores de Gandía. \\ (2) I.F.P. «El Cabañal», Valencia. \\ (3) ICE Universidad Politécnica de Valencia.
}

\section{SUMMARY}

A constructivist view of science learning involves the overall knowledge of cultural factors wich can affect this process. The new trends in the theoretical aspects agree with the acceptance of the interactive nature of the languagethinking relationship. Simultaneously, some research papers show the relationship between language and culturaI surroundings. In this paper we try to establish the experimental justification of the relevance of semantic analysis of the pupil's language and to test some hypotheses about the language role in concept making.

\section{INTRODUCCIóN}

1.1. E] lenguaje en una visión constructivista del aprendizaje

Una aproximación constructivista al análisis de la adquisición de los conceptos científicos implica renocer que el aprendizaje tiene lugar en un marco físico y so- ciocultural determinado que proporciona al alumno un conjunto de percepciones, experiencias personales y significados transmitidos por el ambiente cultural. Éste comprendería tanto el ámbito escolar como las costumbres, técnicas cotidianas, medios de comunicación, etc. No hay que olvidar, por otra parte, que este conjunto 
de experiencias es también determinado por las aspiraciones y actitudes transmitidas en el seno del grupo social en que se desenvuelve el alumno.

Ahora bien, desde el punto de vista del diseño de las actividades de aprendizaje que constituyen el curriculo, necesitamos una metodologia que nos permita caracterizar los rasgos relevantes que dicho ambiente cultural posee en cuanto a la construcción de los conceptos cientificos a través de los diferentes procesos cognitivos.

En diferentes planteamientos teóricos y líneas de investigación, descritos en anteriores trabajos (Llorens $1987 \mathrm{~b}, 1987 \mathrm{c}$ ), puede apreciarse un punto de convergencia a la hora de asignar al lenguaje el carácter de "observable» en el estudio de diferentes procesos que mediante la interacción entre los factores ambientales y la estructura cognitiva de los alumnos conducen a la construcción de los conceptos científicos. El análisis del uso del lenguaje como concreción de las influencias culturales en el aprendizaje aparece ya en Whorf (1971). Dentro del terreno específico del aprendizaje de las ciencias, los trabajos de Mori (1976) y Lynch (1985) muestran, en diversos campos de la Física y Química, cómo la construcción de determinados conceptos se hala profundamente relacionada con las peculiaridades linguísticas de cada idioma. Al mismo tiempo, determinados significados y construcciones verbales evidencian la notable interacción entre el ambiente cultural y el aprendizaje escolar (Bensaude 1982, Solomon 1987). Dentro de otras líneas de investigación se aprecia también ese carácter de "observable» que se asigna al lenguaje. Por ejemplo, Johnstone y Moynihan (1985) utilizan técnicas de asociación de palabras para la elucidacion de mapas conceptuales. Lawson y Sepherd (1976) han observado experimentalmente una correlación significativa entre el grado de complejidad sintáctica de las producciones verbales de los alumnos y su nivel de pensamiento formal.

$\mathrm{El}$ análisis de toda esta información a la luz de las actuales aportaciones teóricas sobre la relación entre pensamiento y lenguaje (Llorens $1987 \mathrm{a}, 1987 \mathrm{c}$ ) permite, a nuestro juicio, no sólo encontrar sentido a esa función del lenguaje como uobservable" de los procesos cognitivos, a la que anteriormente aludíamos, sino también, y quizás sea lo más importante, emplear el propio lenguaje del alumno como un valioso instrumento para intervenir en dichos procesos y orientar adecuadamente la construcción de conceptos. Pasaremos a continuación a aclarar y justificar teóricamente esta propuesta.

Hablar de las influencias culturales en el aprendizaje admite diversos niveles de generalidad. Desde influencias ligadas, por ejemplo, a aspectos tales como los roles asignados por el mundo occidental a los distintos sexos, hasta características muy peculiares del entorno más próximo al alumno. Considerando estas últimas de notable importancia para la concreción de un currículo en actuaciones didácticas determinadas, de- finiremos, a modo de constructo teórico básico y eje vertebrador de nuestra propuesta, el concepto de «microcultura» como "conjunto de experiencias o prácticas cotidianas, ideas transmitidas por el ambiente familiar, social o por los medios de comunicación, susceptibles de interaccionar con el aprendizaje escolar en la construcción de un núcleo específico de conceptos, en una situación espacio-temporal definida» (Llorens 1987 c). Justificaremos a continuacion como la introducción de este concepto responde claramente a una concepción constructivista del aprendizaje.

Una característica inherente a las citadas «microculturas» es su notable variabilidad y dinamismo, cualidades que trasladadas al terreno del lenguaje se traducen en una concepción del significado como algo fluctuante y dinámico, más definido por el uso de la palabra que por su referencia a una determinada clase de entidades (Hanfling, 1980). Esta orientación concuerda con la denominada "visión accional» de la naturaleza de los conceptos, que según Gilbert y Watts (1983) es la más próxima a una concepción constructivista del aprendizaje. Su origen puede situarse en la obra de Kelly (1963), quien concibe la conceptualización como un proceso activo, constructivo e intencional. Freyberg y Osborne (1981) subrayan la función del concepto como un instrumento para organizar nuestra experiencia. Según esta visión, la estructura cognitiva del que aprende sufre una reestructuración continua, ya que cada nueva influencia la modifica. Piatelli-Palmarini (1980) la denomina, a título de analogía, modelo «llama», frente al modelo "cristal» referido a la visión clásica de la naturaleza de los conceptos. Un programa de investigación consecuente con estos planteamientos tiene como objetivo, más que la búsqueda de invariantes o de características universales del desarrollo conceptual, la caracterización y descripción de dominios locales de comprensión o "mini-teorias» (Claxton 1984), Ciencia de los alumnos (Gilbert 1982), o «Esquemas conceptuales alternativos» (Driver 1981). El conjunto de condicionamientos que en una situación dada hacen surgir estas pequeñas elaboraciones teóricas de los alumnos serian precisamente las «microculturas» que anteriormente definíamos.

Retomando la idea del lenguaje como «observable» de los procesos cognitivos, consideramos que analizar la variabilidad semántica del uso de los términos por los alumnos, en los contextos cotidiano y científico, y en la interacción entre ambos, puede constituir una aproximación valiosa a sus esquemas conceptuales alternativos y, más importante todavia, sugerir ideas acerca de cómo éstos pueden evolucionar. Al mismo tiempo, y tal como ya hemos seftalado, el lenguaje puede ser una via privilegiada de actuación sobre la estructura cognitiva del alumno y un modo de explotar el medio cultural del alumno como recurso didáctico.

La importancia de este análisis viene dada, principalmente, porque podemos concebir el aprendizaje como 
algo susceptible de ser representado por procesos de diferenciación y globalización semánticos. En efecto, siguiendo las ideas de Vygotsky (1972), dentro de un contexto cotidiano, los significados evolucionan de lo concreto a lo abstracto, mientras que en el ámbito escolar, conceptos más abstractos e inclusivos sirven para aprehender posteriormente aspectos concretos de la realidad. Ahora bien, en el aprendizaje de las ciencias, y sobre todo en sus niveles más elementales, la delimitación entre conceptos cotidianos y cientificos es notablemente dificil, y la mayor parte de ellos poseen significados en ambos contextos, significados que, además, coexisten en los alumnos, haciendo que la construcción de un concepto no pueda estudiarse como un proceso lineal de generalización o concreción. Coherentemente con los planteamientos teóricos expuestos, el desarrollo de un concepto científico se asemejaría más bien al abandono e incorporación de nuevos significados y a la evolución de las interconexiones entre ellos. Estas consideraciones nos obligan a detenernos en un análisis más detallado del problema de la polisemia de los términos utilizados en las ciencias.

\subsection{Lenguaje científico y su relación con el ordinario}

El diagrama expuesto en la fig. 1 es una clasificación de las palabras en base a sus relaciones semánticas con la actividad científica. Nos servirá como hilo conductor del análisis que vamos a realizar:

\section{figura I}

Contextos cientifico y ordinario en el uso del lenguaje.

Viscabulatio appleato:

Sigałfeado destro del conterto

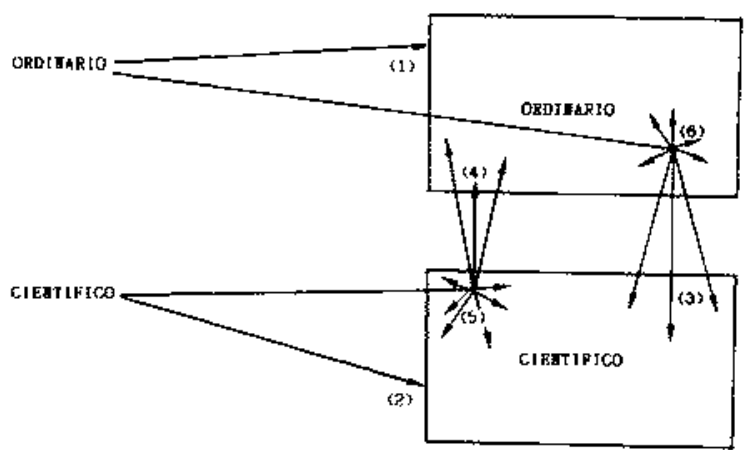

a) (1) representa el conjunto de palabras del lenguaje ordinario entre cuyos significados no hay ninguno relativo a la actividad científica; (2), por el contrario, se refiere a aquellos términos cuyo uso está completamente restringido al ámbito científico («entalpia», "eutéctico», "nucleofílico», etc.); (1) y (2) representan un primer nivel de diferenciación entre lenguaje científico y el ordinario.

b) No obstante, la situación no suele ser tan sencilla. La mayor parte de la terminología científica utilizada en la enseñanza básica y secundaria procede del lenguaje ordinario, tras un complejo proceso de diferen- ciación y precisión semántica de los significados etimologicos o del uso ordinario. Vienen representados en (3). La relación entre significados científicos y ordinarios en este grupo puede ser muy variada: desde términos como «polar», "fase», "reductor», en los que el significado científico dista considerablemente del ordinario, a otros en que ambas acepciones son más cer. canas: «enlace», «equilibrio»...

c) En aquellos dominios científicos de:

- Mayor impacto socioeconomico.

- Que son objeto preferente de divulgación en los medios de comunicación.

- Que incluyen términos adecuados para establecer analogías con otros campos del conocimiento.

Ciertos términos pueden pasar al lenguaje ordinario (4) conservando en mayor o menor medida su significado inicial.

Un caso típico es el término «átomo», cuyos derivados "atomístico», "atomizar», etc., son corrientemente empleados en contextos extracientíficos. Podemos ci. tar también «ósmosis», que se emplea de modo analógico al referirse a la influencia mútua entre personas o grupos sociales, o "espectro», que se utiliza ordinariamente para aludir a la disociación de cualquier estado complejo en los estados simples que lo componen.

d) Por último, nos referiremos a la diferenciación semántica dentro de los propios contextos científicos y ordinario. Nos referiremos, en primer lugar, al científico (5). Evidentemente, la persistencia histórica de algunas palabras no implica una continuidad conceptual. La palabra "átomo» no significa lo mismo en la Filosofía Natural griega que en la Química moderna. Ni la palabra «elemento» en la obra de Aristóteles que en la Química de Lavoisier. Sobre términos como "oxi* dación» o "ácido» podríamos hacer una reflexión similar.

La diferenciación semántica dentro del contexto científico no obedece únicamente a razones históricas. Tal es el caso de «actividad», término que dentro del contexto químico puede referirse a la mayor o menor tendencia de una substancia a experimentar cambios químicos en una situación determinada (acepción más próxima a la ordinaria) o bien puede referirse, en la Termodinámica química, a los coeficientes de actividad.

Quedando fuera de nuestra atención, procesos similares tienen lugar en el seno del lenguaje ordinario (6).

Esta clasificación de las relaciones entre contexto cotidiano y científico la juzgamos en un marco de referencia útil a la hora de estudiar los problemas inherentes al uso de la terminología por los alumnos.

\section{HIPÓTESIS DE TRABAJO}

La permanente alusión a la naturaleza del lenguaje co. mo «observable» de los procesos cognitivos ha sido en 
realidad una hipótesis subyacente en todo nuestro planteamiento teórico. La constatación de esta hipótesis ha sido el objeto de diferentes investigaciones de tipo descriptivo ya expuestas en un anterior trabajo (Llorens 1987a). Tan sólo señalaremos algunos ejemplos fruto de trabajos posteriores y que creemos relevantes. Ello constituirá el primer bloque de experiencias que describiremos.

La aceptación de la anterior hipótesis nos justifica metodológicamente para utilizar la diferenciación semántica (entre contexto cotidiano y contexto escolar, y en el seno de cada uno de ellos, (fig. 1) como variable en el estudio de la influencia del lenguaje en la construcción de conceptos. Ello nos permite abordar la contrastación de dos hipótesis de particular interés:

A) Dado el carácter de organizador de la experiencia que las tendencias teóricas actuales asignan al lenguaje, el dominio del vocabulario correspondiente a los prerrequisitos del aprendizaje de una teoría científica se hallará notablemente relacionado con la capacidad de elaborar adecuadamente los conceptos de dicha teoría a partir de los hechos experimentales. Esta relación presentará además una especificidad en cuanto a contenidos.

En nuestro caso concreto, se estudia la construcción de los conceptos químicos necesarios para el establecimiento de la teoria atómico-molecular a partir de los hechos experimentales que apoyan su propuesta. Su nivel de adquisición se relaciona con el nivel de precisión semántica en el empleo de vocabulario representativo de los prerrequisitos de aprendizaje de dicha teoría. Desde el punto de vista estadístico, la técnica utilizada para evaluar dichas relaciones es el cálculo de coeficientes de correlación parcial eliminando el efecto de aquéllas que pudieran hacer falsas las citadas correlaciones.

B) En íntima relación con la hipótesis anterior, la presencia de determinados significados cotidianos podrá dificultar la construcción de los conceptos científicos. Este efecto se estudia en relación a masa, volumen y densidad, a través de problemas relacionados con la conservación de la masa y con el principio de Arquímides.

\section{EXPERIENCIAS REALIZADAS}

\subsection{De carácter descriptivo}

Comentaremos tan solo algunos casos representativos. Es interesante, por ejemplo, observar la diversidad de conexiones que una palabra como "energía» es capaz de generar en un grupo de 18 aiumnos de 14-16 años, aplicando técnicas de asociación de ideas (Llorens 1987b):

También es interesante observar, en relación al concepto introducido de "microcultura», cómo un ámbito tan concreto de la experiencia del alumno como el mundo de la motocicleta puede generar nuevos $-\mathrm{y}$ potencialmente relevantes desde el punto de vista del aprendizaje - significados para algunos términos cientificos:

figura 2

Conexiones semánticas del término "energía» en un grupo de alumnos de 14-16 años.

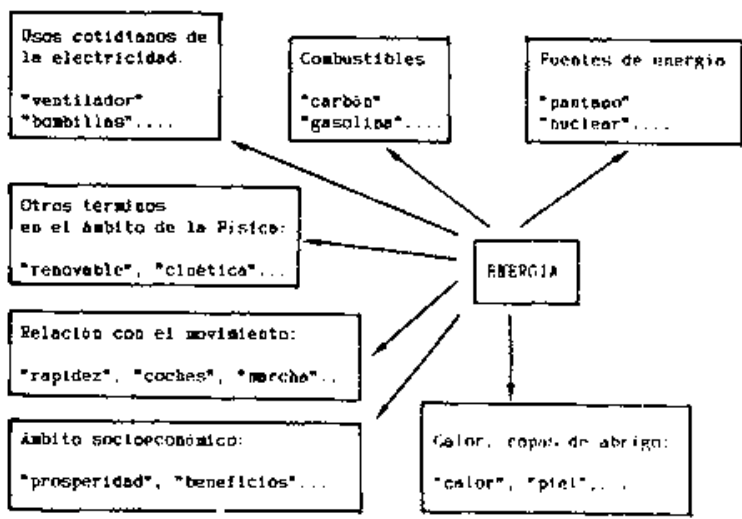

figura 3

L.a significación de algunos términos cientificos en un contexto concreto: el mundo de la motocicleta.

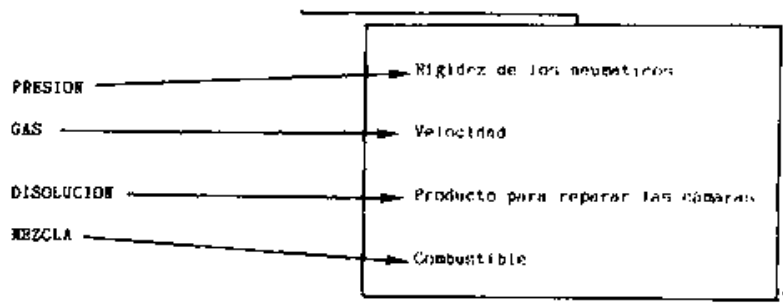

Empleando métodos basados en la asociación palabra. imagen (Llorens, 1987a) pueden apreciarse las diferentes representaciones que términos como "reaccion"», "gas», "difusión», «transformación» generan en los alumnos (primer grado de FP, 14-16 anos).

figura 4

Diferentes representaciones del tétmino «Reacción».

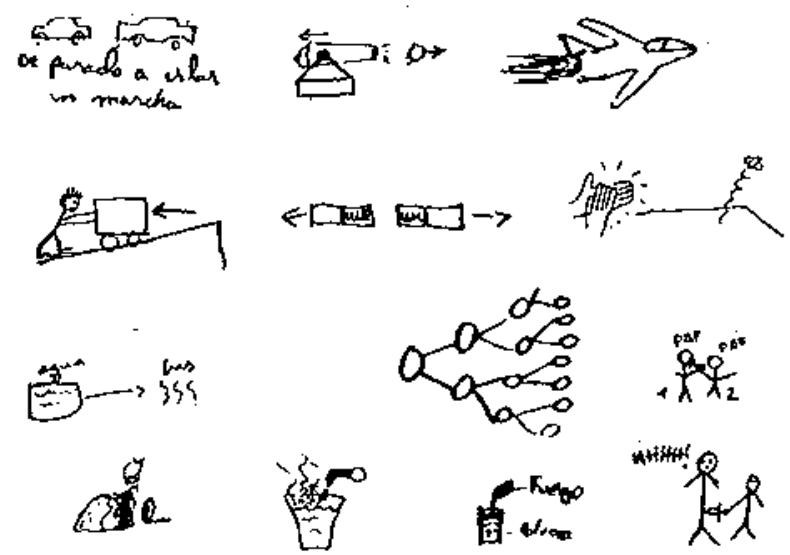

ENSEÑANZA D̀E LAS CIENCIAS, 1989, 7(2) 
En otro cuestionario similar, en el que se proporcionaba a los alumnos un conjunto de representaciones que debfan asociar a una lista de términos (Llorens 1988a), se observa como las relaciones reflejan errores conceptuales típicos.

figura 5

«Dilatación».
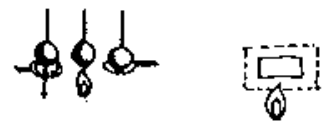

18

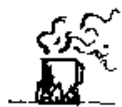

153
"Cambio de Estado"
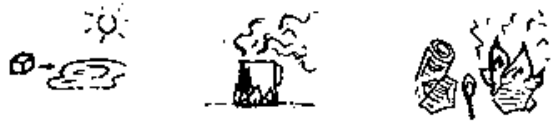

$7 \geq 2$

$3 \times 1$
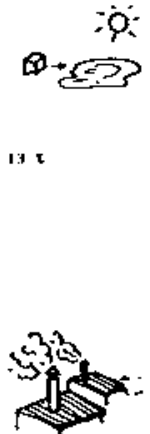

$13 x$

$1 \div$

Estas tendencias erróneas pueden ser caracterizadas por el empleo de un determinado campo semántico. En la figura 6 se observan representaciones, realizadas por alumnos de 14-16 años, de la dilatación de un gas junto a algunos de los términos utilizados en ítems de frases incompletas sobre este mismo aspecto:

\section{figura 6}

1TEM. Cuando calentanos un tubo de ensayo ton aire al que le henos colocado un globo en la bora, sucede lo que auestra la tigura:

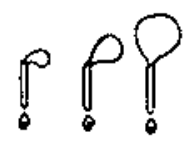

Derinos que al are se ha.........

Aigunas respuestas:

$$
\begin{aligned}
& \text { DESPLA?ROOO } \\
& \text { ELEUAOD } \\
& \text { TRASLAOADD } \\
& \text { SUBIOD }
\end{aligned}
$$
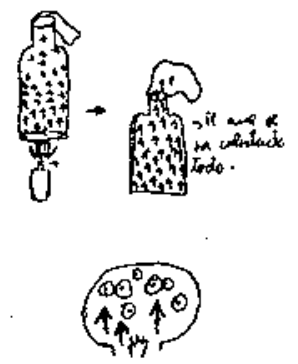

Esta diversidad de representaciones junto al escaso dominio de las estructuras gramaticales puede redundar en una escasa capacidad de comunicación por parte del alumno, Como ejemplo, podemos observar en la figura 7 las «transformaciones» llevadas a cabo cuando se les pide describir verbalmente un montaje sencillo, y a otros alumnos reconstruir éste a partir de las descripciones verbales de los compañeros.

figura 7

Dî́erentes interpretaciones de la descripción escrita de una figura:
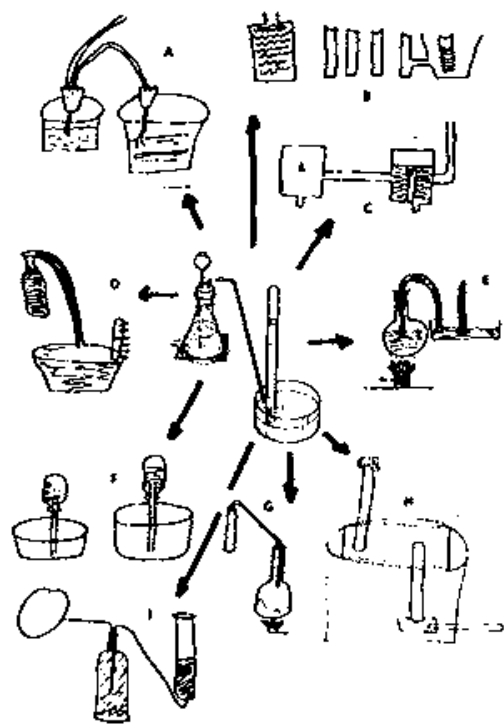

3.2. Experiencias encaminadas a la contrastación de las hipótesis

3.2.1. Relación entre precisión semóntica en el uso del lenguaje preteórico y grado de adquisición de los conceptos básicos de la teoría atómico-molecular en un contexto experimental

Una descripción detallada de esta experiencia puede encontrarse en la Tesis Doctoral del autor (Llorens 1987a). Tan solo haremos aquí referencia a los aspectos más esenciales.

A partir de la información obtenida en una primera fase, basada en el empleo de cuestionarios abiertos y entrevistas, en un conjunto de unos 800 alumnos de FP

\section{figura 8}

Diagrama conceptual en que se basa el diseño de la investigación.

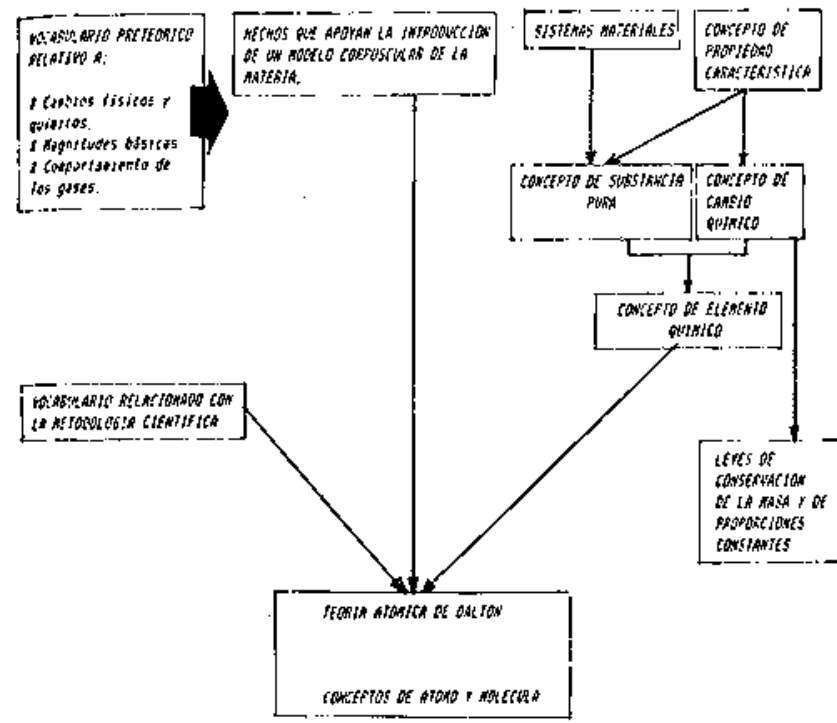


y BUP de 11 centros de la Comunidad Valenciana, se diseñó una segunda fase compuesta por la aplicación. de cuatro pruebas estructuradas en torno al siguiente diagrama conceptual:

La primera prueba constaba de 11 f́tems de opción múltiple asociados a seis experiencias grabadas en video, técnica cuya idoneidad ya había sido contrastada en otras investigaciones (Oliver y Roberts 1980 y Ben Zvi, Hofstein y Kempa 1982). Su contenido giraba en torno a:

- Naturaleza del cambio químico.

- - Cambio en la naturaleza de las substancias.

- Confusión con cambios fisicos.

- Conservación de la masa en procesos en que intervienen gases.

- Naturaleza corpuscular de la materia y modelo cinético-molecular.

- Difusión de gases.

- Disolución.

La segunda y tercera constaban respectivamente de 9 y 18 items en torno a:

- Conservación de la masa en procesos físicos y químicos.

- Interpretación del comportamiento de los gases en base a un modelo corpuscular de la materia.

- Definición operativa de los sistemas materiales a par. tir de los procesos de separación.

- Terminologia relativa a la definición de los sitemas materiales.

- Terminología relativa a los métodos de la Ciencia.

- Conceptos de átomo y molécula y su relación con los de elemento y compuesto.

Las alternativas erroneas de todos estos ítems fueron extraidas de las investigaciones realizadas en la fase preliminar.

La prueba 4 constaba de 30 frases incompletas en las que se habia eliminado una palabra que debía ser propuesta por el alumno. Los aspectos estudiados fueron:

- Cambios físicos y químicos (terminología de los cambios de estado, disolución, cambios químicos, etc.).

- Terminología de las leyes del cambio químico (términos "conservación» y "proporción»).

- Terminología relativa al comportamiento de los gases.

- Terminología relativa a los métodos de la ciencia.

- Terminología relativa a las magnitudes básicas (masa, volumen...).

Aspectos que constituyen el núcleo de prerrequisitos de la teoría atómica, descrito en la fig. 8.

El conjunto de estas pruebas fue aplicado en dos sesiones de 45 minutos, a 606 alumnos de $2^{\circ}$ de BUP y primer grado de FP, de 6 centros de la Comunidad Va- lenciana, antes de abordar la enseffanza de los temas de Química.

El método general de trabajo fue constituir escalas aditivas a partir de estos ítems que tuvieran una unidad de significado, procediendo a su corrección por el método del coeficiente de Cronbach para eliminar aquelos ítems cuya presencia dismiruye su consistencia interna. El paquete informático utilizado en el conjunto de la investigación fue la última versión del S.P.S.S. instalada en el C.I.U.V.

En la constrastación de la hipótesis que nos ocupa las escalas empleadas fueron:

NCQ Naturaleza del cambio químico.

CMASAG Conservación de la masa en procesos en que intervienen gases.

NCM Naturaleza corpuscular de la materia.

CSM Clasificación de los sistemas materiales.

LCQ Leyes del cambio químico.

TCFISQUI Terminologia de los cambios físicos y químicos.

TGASES Terminología relativa al comportamiento y leyes de gases.

TCSM Terminología relativa a la clasificación de los sistemas materiales.

TLCQ Terminología de las leyes del cambio químico.

TMCF Terminología de los métodos científicos.

En la fig. 9 aparecen los coeficientes de correlación parcial entre estas escalas utilizando como variables de control la calificación global del curso anterior, la edad, la actitud -evaluada en un cuestionario anexo-e el tipo de estudios (BUP-FP) y la repetición o no de curso. En todos los casos, en cuanto a la significación de los coeficientes de correlación, siendo 599 el número de grados de libertad, $\mathrm{P} \leqslant 0.001$.

figura 9

Correlaciones parciales entre las escalas definidas.

\begin{tabular}{|lccccc|}
\hline & TCPISQUI & TGASES & TCSI & TLCQ & TXCP \\
ICO & 0.1894 & 0.1844 & 0.1024 & 0.1155 & 0.1923 \\
CXASAG & 0.3153 & 0.2894 & 0.1765 & 0.1745 & 0.2587 \\
ICM & 0.2471 & 0.2236 & 0.1501 & 0.1889 & 0.1957 \\
CSI & 0.1976 & 0.1601 & 0.1593 & 0.1344 & 0.1586 \\
LCQ & 0.2642 & 0.2177 & 0.1340 & 0.1976 & 0.2156 \\
\hline
\end{tabular}

Los resultados muestran cómo la precisión en el uso de la terminología básica se halla asociada a un mejor nivel de adquisición de conceptos a partir de los hechos experimentales. Esta asociación presenta una notable especificidad en cuanto a contenidos y cabe des. tacar la relevante interconexión entre el empleo correcto de la terminología propia de los métodos de la Ciencia 
y la interpretación correcta de hechos experimentales. Trasladando este lenguaje a un terreno más epistemológico, diriamos que el dominio del lenguaje preteorico es un fiel exponente del grado en que la observación y percepción de los hechos experimentales son condicionados por la presencia de esquemas teóricos en el alumno.

\subsubsection{Influencia de algunos significados cotidianos en la adquisición de conceptos}

Se estudió, mediante una prueba de asociación de palabras y formación de frases (Llorens $1987 \mathrm{~b}$ ), la relación entre la presencia de acepciones ordinarias potencialmente susceptibles de generar problemas en la construcción de los conceptos de masa, volumen y densidad con su grado de adquisición evaluado a través de problemas relacionados con la conservación de la masa y el principio de Arquímedes (ver anexo).

La experiencia se llevó a cabo con 91 alumnos pertenecientes a un grupo de $8^{\circ}$ de EGB, $2^{\circ}$ de FP 1 y $1^{\circ}$ de FP 2. Mediante la primera de las pruebas este conjunto fue subdividido en dos, según aparecieran o no algunas de las acepciones expuestas en la fig. 12, en dos o en los tres têrminos. Entre ambos subgrupos se aplicó la prueba de la «t» de Student a las puntuaciones obtenidas en los problemas relacionados con la conservación de la masa y con el principio de Arquímedes, respectivamente.

En la fig. 11 aparecen Ios resultados obtenidos:

En ellos se observa cómo en el primero de los problemas existe una clara influencia de la presencia de las acepciones cotidianas consideradas problemáticas.

figura 10

Acepciones identificadas en el empleo de los términos «Masa», "Voumen» y "Densidad».
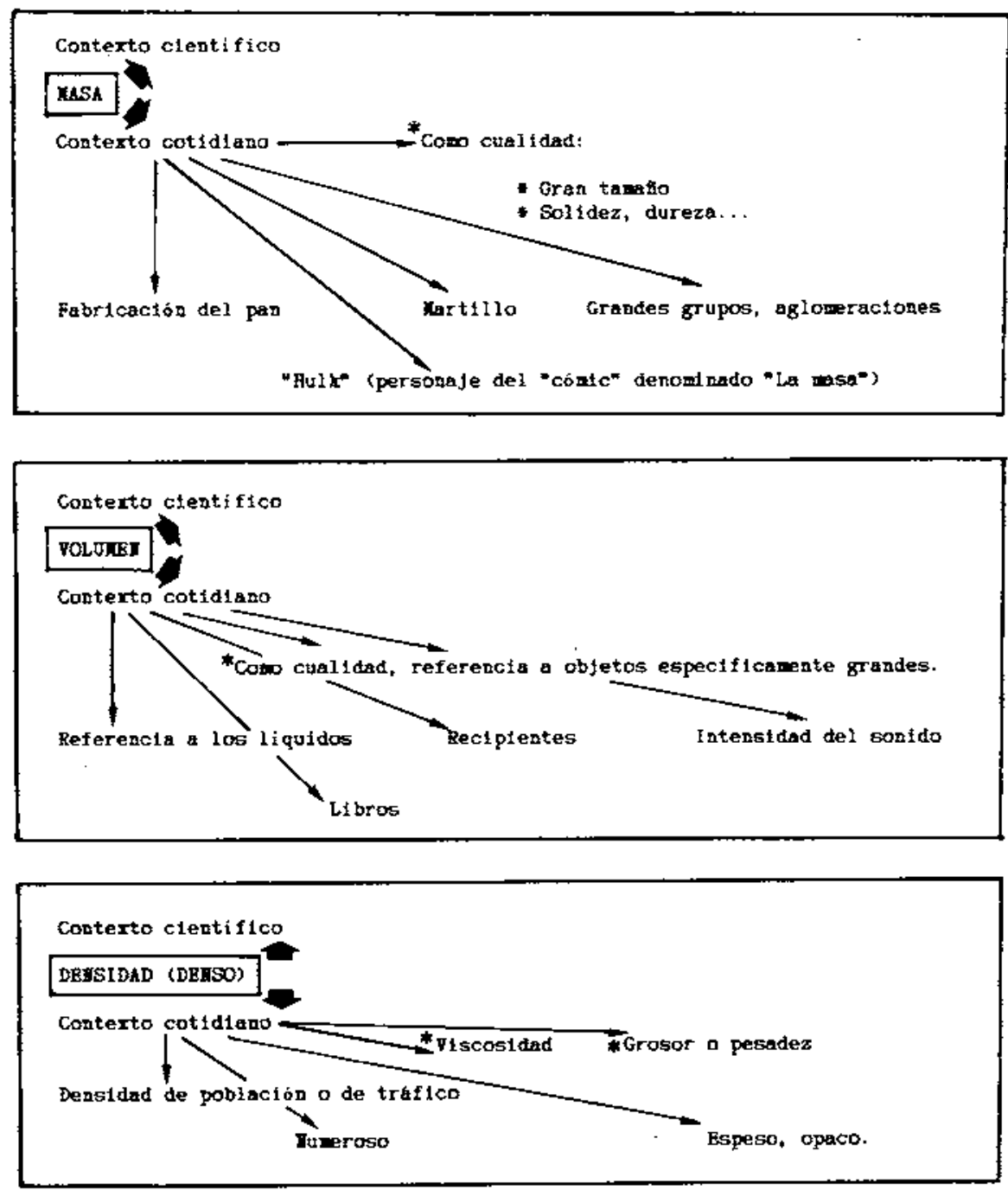


\section{figura 11}

Prueba de la «t» de Student para verificar la relación entre la presencia de ciertas acepciones cotidianas y la comprensión de los conceptos de masa, volumen y densidad en un contexto experimental.

Grupo A: Presencia de al menos dos de las acepciones cotidianas señaladas (*) en la fig. 10 .

Grupo B: No presencia.

\begin{tabular}{|c|c|c|c|c|}
\hline \multirow{3}{*}{$\begin{array}{l}\text { Puotuacitio } \\
\text { Conservectón } \\
\text { wsa }\end{array}$} & \multicolumn{2}{|c|}{ YaJores adsos } & $" z$ & $a$ \\
\hline & Grupo \& & Grupa $\#$ & & \\
\hline & 2.33 & 3,10 & 2,52 & 0,02 \\
\hline $\begin{array}{l}\text { Priociplo de } \\
\text { trqui edes }\end{array}$ & 0.57 & 1,31 & 1,00 & 0.10 \\
\hline
\end{tabular}

\section{CONCLUSIONES}

Los resultados de esta investigación conllevan, a nuestro juicio, un conjunto de implicaciones de indudable interés, máxime en un momento como el actual, en que parece gestarse una profunda reforma del sistema educativo:

- Los puntos de referencia teóricos de los que parte nuestra investigación muestran una clara evidencia experimental, to cual trae dos claras consecuencias:

- La necesidad de una mayor atención al lenguaje en la investigación didáctica de orientación constructivista.

\section{REFERENCIAS BIBLIOGRÁFICAS}

BEN ZVI, 1976. The Effectiveness of Filmed Experiments in High School Chemical Education. Journal of Chemical Education, Vol. 53, p. 518.

BENSAUDE, B., 1982. Resultats d'une enquête sur quelques yocables d'orientation scientifique. (LIRESPT, E.N.S. Fontenay, E.N.S. St. Cloud: Paris).

CLAXTON, G.L., 1984. Teaching and acquiring scientific knowledge. (En Keen, $\mathrm{T}$. y Pope, M. (eds.) Kelly in the classroom: Educational Applications of Personal Construct Psychology). (Cybersystems: Montreal).

DRIVER, R., 1986. Psicologia cognoscitiva y esquemas conceptuales de los alumnos. Enseñanza de las ciencias, Vol. 4 (1), pp. 3-15.

FREYBERG, P. y OSBORNE, R.J., 1981. Who structures the curriculum: teacher or learner? SET Research Information for Teachers 2, item 6.

GILBERT, K.J. y WATTS, M.D., 1983. Conceptions, misconceptions and alternative conceptions: Changing perspectives in Science Education. Studies in Science Education, Vol. 10. pp. 61-98.

HANFLING, O., 1982. Filosofia del lenguaje. (ICE Universidad de Valencia - Open University, Valencia).

JOHNSTONE, A.H. y MOYNIHAN, T.F., 1985. The realtionship between performances in word association tests and achievement in Chemistry. European Journal of Science Education, Vol. 7, (1), pp. 57-66.
- La posible conveniencia de impulsar una línea de investigación fundamentada en el análisis de los problemas lingüisticos en el diseño de actividades de aprendizaje, como, por ejemplo, el uso intercontextual de la terminología.

- La función del lenguaje como expresión de la incidencia de aspectos culturales en el aprendizaje creemos que es extraordinariamente relevante, pues puede permitir la caracterización de influencias ambientales sobre las cuales descansa la necesaria diferenciación curricular que exige la implantación de una enseñanza comprensiva hasta los 16 años.

- Dentro del terreno de diagnostico de difícultades de aprendizaje y caracterización de aspectos «microculturales» en los diferentes colectivos de alumnos, debemos destacar la posible utilidad de las técnicas introducidas, pues proporcionan abundante informacion, siendo rápidas y sencillas de aplicar.

- Como posibles investigaciones dentro de esta línea podemos proponer el estudio evolutivo de los significados en un determinado núcleo conceptual, la influencia de las actividades de reflextón lingüística en el rendimiento del aprendizaje, la caracterización de influencias culturales y de los significados del lenguaje ordinario en la construcción de determinados conceptos científicos, etc.

KELLY, G.A., 1963. The Psychology of Personals Constructs. (W.W. Norton: New York).

LAWSON, A.E. y SEPHERD, G.D., 1976. Syntactical Complexity in Written Language and Cognitive Development at the Formal Level. Science Education, Vol. 63 (1), pp. 73-81.

LYNCH, P.P., 1985. The Language of Science in the High School Student: The Recognition of Concept Definitions: A Comparison between Hindi Speaking Students in India and English Speaking Students in Australia. Journal of Research in Science Teaching, Vol. 22 (7), pp. 675-686.

LLORENS, J.A., 1987. Propuesta y aplicación de una metodologia para analizar la adquisición de los conceptos químicos necesarios en la introducción a la teoría atomicomolecular: Percepción de los hechos experimentales, sus representaciones y el uso del lenguaje en alumnos de Formación Profesional y Bachillerato. Tesis Doctoral no publicada, Universidad de Valencia.

LLORENS, J.A., 1987. El uso de la terminología científica en los alumnos que comienzan el estudio de la Quimica en la Ensernanza Media. Una propuesta metodológica para su análisis. Enseñanza de las Ciencias. Vol., 5 (1), pp. 33-40.

LLORENS, J.A., 1987. El medio cultural y la adquisición de los conceptos científicos. Infancia y Aprendizaje, Vol. 39-40, pp. $47-55$. 
MORI, I., 1976. The Effect of Language on a Child's Conceptions of Speed: A comparative Study on Japanese and Thai Children. Science Education, Vol. 60 (4), pp. 532-534.

OLIVER, P.M. y ROBERTS, I.F., 1980. Filmed Sequences and Multiplechoice tests. Education in Chemistry, pp. 132-133.

PIATELLI-PALMARINI, M., 1980, Language and Learning: The debate between Jean Piaet and Noam
Chomsky. (Routledge and Keagan, P.: London).

SOLOMON, J., 1987. Social Influences on the Construction of Pupil's Understanding of Science. Studies in Science Education, Vol. 14, pp. 63-82.

VYGOTSKY, L.S., 1962. Thought and Language. (M.I.T. Press: Cambridge).

WHORF, B.L., 1971: Language, Thought and Reality. (M.I.T. Press.: Cambridge).

\section{ANEXO \\ CUESTIONARIO SOBRE LA ADQUISICIÓN DE LOS CONCEPTOS DE MASA, VOLUMEN Y DENSIDAD}

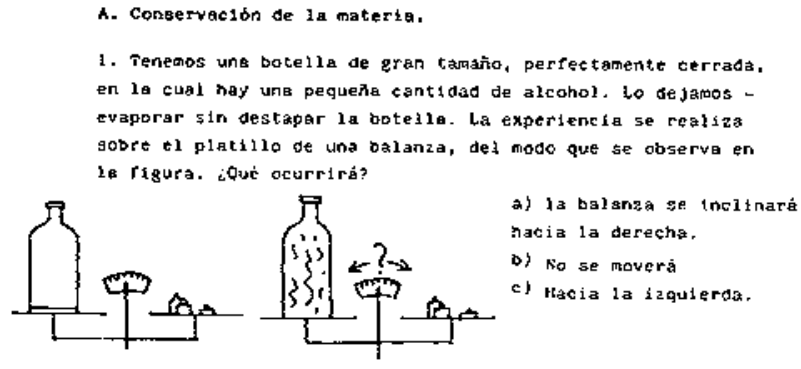

2. Kabrís observaoo que el intelo floka en agua. S1 ponemos un cubito de hielo en el platillo de una balaiza y la equilibramos con pesas ¿Qué ocurrírá al derretirse el hielo?

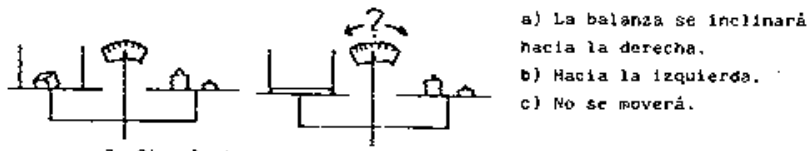

3. Si calentamos un tubo de ensayo con un globo en la boca, tal como se observa an la plgura, ocutrc esto:

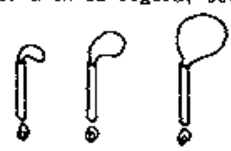

¿ha aumentado la cantidad de aire?

a) $\$ 1$ b) No

c) No. simplemente el aire que habla en tl tubo se ha trasladado al globo.

4. Si en el bombin de una blciclcta tapamos la sallda, podemos comprimitr el alre que contlene. ¿En qué caso hay mág al re?

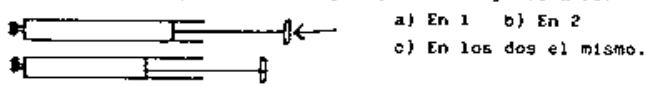

5. Cuando cslentsmos un objeto awanta de tansho. Por elso natords v1sto que, por ejemplo, ae de je uns pequeß̄⿴ separación entre los tramos del rall del tren. 51 tenemos una plezs metślica y la volvemos a poner en 1 platillo ise inelinarás la balanza?

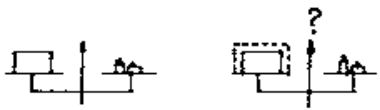

a) \$1, puts a más volumen. más magá.

b) ro

c) S1, pero nacie la derecha, pues al dilatarse, su masa ol sminuye.
B. Principlo de Arquinedeo

1. Tenenos dos bloqucs exactarente 1guales, uno de plomo y otro de aluminio:
is at.

Los introducimos en dos reciplentes que t1enen la alsma cantidad de agua.

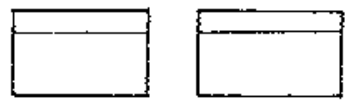

iHabrá direrenela en el nivel alcanzado por el agua en ambos recipientes?

$$
\begin{aligned}
& \text { a) Ho. puts los dos bloques } \\
& \text { trenen el misalo volumen. } \\
& \text { b) } 51 \text {, pues bunque tienen el } \\
& \text { mismo volumen, se trata de } \\
& \text { difercntes substancias. } \\
& \text { c) Dependerá de cómo sean } \\
& \text { los reciprences. }
\end{aligned}
$$
pesa mucho más que el alflier icomo 10 cxpllicas?

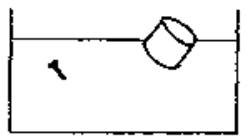

a) El alfiler tiene punta. b) El bote tiene alre en st interior y su relación $M / V$ eq menor que en el alfiler. c) Todo to nueco rlota

3. ¿Por qué un globo lleno de eire carliente se tleva?

Q) Porque sus moléculss asciencen nacia artiba.

b) Porque su masa of smirinye

c) Porque diminuye su dengldad, siendo menor que la del aire que 10 rodea. 